\title{
Resistive and reactive loads influences on highly coupled piezoelectric generators for wideband vibrations energy harvesting
}

\author{
Adrien Morel ${ }^{* a, b}$, Adrien Badel $^{\mathrm{b}}$, Romain Grézaud ${ }^{\mathrm{a}}$, Pierre \\ Gasnier $^{\mathrm{a}}$, Ghislain Despesse ${ }^{\mathrm{a}}$, Gaël Pillonnet ${ }^{\mathrm{a}}$ \\ ${ }^{a}$ Univ. Grenoble Alpes, CEA LETI, 38000 Grenoble, France \\ ${ }^{\mathrm{b}}$ Univ. Savoie Mont Blanc, SYMME, 74000 Annecy, France
}

\section{Abstract}

One of the main challenges in energy harvesting from ambient vibrations is to find efficient ways to scavenge the energy, not only at the mechanical system resonance, but on a wider frequency band. Instead of tuning the mechanical part of the system, as usually proposed in the state of the art, this paper develops extensively the possibility to tune the properties of the harvester using the electrical interface. Due to the progress in materials, piezoelectric harvesters can exhibit relatively high electromechanical coupling: hence, the electrical part can now have a substantial influence on the global parameters of the piezoelectric system. In order to harness the energy efficiently from this kind of generator on a wide frequency band, not only the electrical load's effect on the harvester's damping should be tuned, but also its effect on the harvester's stiffness. In this paper, we present an analytical analysis of the influences of the resistive and reactive behavior of the electrical interface on highly coupled piezoelectric harvesters. We develop a normalized study of the multiphysic interactions, reducing the number of parameters of the problem to a few physically meaningful variables. The respective influence of each of these variables on the harvesting power has been studied and led us to the optimal electrical damping expression and the influences of the damping and of the coupling on the equivalent admittance of the piezoelectric energy harvester (PEH). Finally, we linked these normalized variables with real reactive load expressions, in order to study how a resistive, capacitive and inductive behaviors could affect the global performances of the system. The theoretical analysis and results are supported by experimental tests on a highly coupled piezoelectric system $\left(k^{2}=23 \%\right)$. Using an adequate tuning of a RC load at each frequency, the maximum harvested power $(11 \mu \mathrm{W})$ under a small acceleration amplitude of $0.5 \mathrm{~m} . \mathrm{s}^{-2}$ is reach over a $14 \mathrm{~Hz}$ large frequency band around $105 \mathrm{~Hz}$ which has been predicted by the model with less than $5 \%$ error.

\section{Keywords}

*Corresponding author, E-mail address: adrien.morel@cea.fr 
Vibration harvesting; Piezoelectricity; Highly coupled generators; Reactive electrical interface; Optimal damping; Resonance.

\section{Introduction}

The last decade has seen a growing interest in new energy scavenging sources that could replace chemical batteries (Schlichting et al., 2012), such as microbial fuel cells (Wanderoild et al., 2018), solar energy harvesters (Raghunathan et al., 2005), or thermal generators (Sodano et al., 2007). Mechanical energy harvesting is a good way to harvest the ambient energy, especially in closed confined environments. Piezoelectric elements are of particular interest because of their high energy densities and integration potential (Caliò et al., 2014). Since there exists a coupling between the electrical domain and the mechanical domain in a piezoelectric harvester, it has been proven that the interface circuit has a certain influence on the global harvesting efficiency of the system (Zhu et al., 2009). During the last two decades, there has been a relatively long-standing interest in strategies and electrical interfaces that could help increasing the scavenged power (Guyomar and Lallart, 2011; Liang, 2017; Wu et al., 2013; Shi et al., 2016)

Ottman et al. and Lesieutre et al. have proposed to model the influence of the electrical interface as a mechanical damper that could be electronically tuned, for instance using a DC/DC converter (Ottman et al., 2002; Lesieutre et al., 2004). In an extensive theoretical study that takes in account the nonlinearities induced by the required $\mathrm{AC} / \mathrm{DC}$ conversion, Shu et al. proved that there exists a particular damper that maximize the power at the resonance frequency (Shu and Lien, 2006).

In 2005, Guyomar et al. proposed new synchronous harvesting circuits based on the commutation of inductive switches synchronously with the mechanical vibration. These highly nonlinear circuits, such as Synchronous Electric Charge Extraction (SECE) or Synchronized Switch Harvesting on Inductor (SSHI), could harvest the energy way more efficiently than the usual DC/DC used to get an optimal damping (Guyomar et al., 2005). These strategies have been widely used and developed on lowly coupled piezoelectric generators or excited out resonance, and they undoubtedly outperformed the standard approach, as they were able to emulate higher electrical damping that were closer to the optimal damping required to reach the maximum harvested power (Lefeuvre et al., 2005). However, it has also been shown that if the electromechanical coupling factor is high enough, the synchronous techniques are no longer a better choice than the traditional harvesting strategy using a DC/DC (Lefeuvre et al., 2006). Indeed, the electrical damping induced by the nonlinear process is getting too important for highly coupled piezoelectric generators, and hence is not optimal (Lefeuvre et al., 2006).

Due to the last progresses in materials science and piezoelectric generator design, the electromechanical coupling factor, initially relatively low, is gradually getting greater (Cho et al., 2005; Badel and Lefeuvre, 2014). This has consequences on the frequency response of PEHs. The optimal electrical load is no longer only resistive and inductive but is resistive and capacitive between the piezoelectric generators short-circuit and open circuit resonant frequencies. As shown in this paper, the higher the coupling, the wider this frequency band, and hence it is increasingly important to include an additional 
tunable capacitive load that has a direct impact on the open circuit resonant frequency of the PEH.

Recently, some researchers have proposed to use this increasing coupling to design interfaces that could harvest the energy efficiently on a wide frequency band, thanks to their reactive and resistive impedance emulation (Badel and Lefeuvre, 2014; Ahmed-Seddik et al., 2013; Hsieh et al., 2015). Indeed, it has been proven that even highly nonlinear electrical strategies can be seen and modelled as combination of linear reactive and resistive loads, thanks to the first harmonic approximation (Brufau-Penella and Puig-Vidal, 2009; Liang and Liao, 2010). While the influences of the resistive (Wu et al., 2006), and capacitive (Liao and Sodano, 2009) parts of the electrical interface have already been analytically studied, to the authors knowledges, there is no thorough analysis gathering all the resistive and reactive influences of the load on highly coupled harvesters $\left(k_{m}^{2}>4 \xi_{m}\right)$ with experimental demonstrations of these influences.
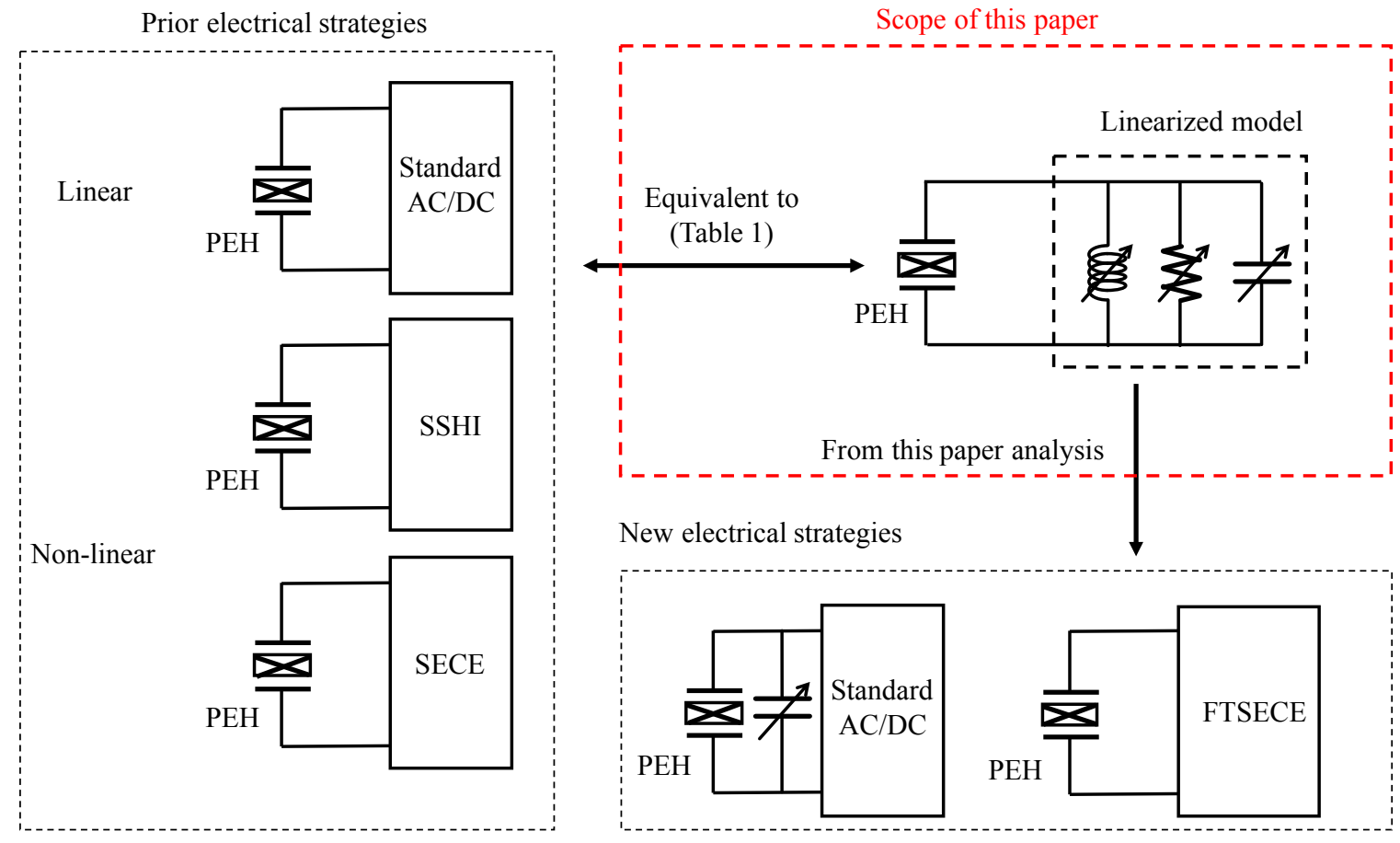

Fig. 1. Scope of this paper

As described in Fig. 1, we propose in this paper a generalized analysis of reactive and resistive electrical loads influences for highly coupled piezoelectric generators. We study the optimal electrical damping, and investigate on the piezoelectric admittance characteristics in order to highlight the influence of the coupling factor and mechanical damping on its resonance and anti-resonance frequencies. We then analyze the influences of the resistive, capacitive and inductive part of the electrical interface on the dynamics of the electromechanical systems, in order to determine the theoretical upper bounds of the harvested power and bandwidth. From these results, we may estimate the maximum power frequency response of any electrical interface, considering that any (nonlinear or not) interface can be modelled as a combination of resistive and reactive loads (Liang and 
Liao, 2010). To do this, we show that the piezoelectric voltage can be split in two terms: an electrical damping and an electrical stiffness. We will then investigate on those terms in order to prove that to maximize the harvested energy in highly coupled systems, not only the electrical damping should be considered, but also the electrical stiffness. Taking these two terms into account allows the harnessing of the maximized power on a large frequency band. In a last section, we validate the proposed approach through experimental tests of resistive and capacitive loads connected to a highly coupled PEH.

\section{PEH modeling and coupling analysis}

\section{Model and fundamental equations}

A piezoelectric energy harvester is used to convert mechanical energy into electrical energy. First, the vibration is transmitted in the host structure in order to stretch the piezoelectric materials. Connecting an interface circuit to the piezoelectric materials allows the electrical charges to flow and hence allows the energy transfer. Due to the backward coupling, it is important to note that the energy transfer is impacted by every stage of conversion:

\section{-The mechanical transfer in the harvester structure \\ -The electromechanical conversion \\ -The electrical energy transfer}

The aim of this paper is to analyze the impact of reactive loads on the electromechanical dynamics of the harvester, and to determine as well the optimal electrical loads that maximize the energy conversion and transfer for highly coupled generators. Considering the generic mechanical-to-electrical conversion model depicted in Fig. 2 and described extensively in (Arroyo et al., 2012), the electromechanical constitutive equations can be written as:

$$
\left\{\begin{array}{c}
M \ddot{x}(t)+D \dot{x}(t)+K_{s c} x(t)+\alpha v_{p}(t)=-M \ddot{y}(t)=-M \gamma \\
i(t)=\alpha \dot{x}(t)-C_{p} \dot{v}_{p}(t)
\end{array}\right.
$$

where $x, y, v_{p}$ and $i$ stand for the dynamic mass displacement, the ambient displacement, the piezoelectric output voltage and current respectively. $M$ is the dynamic mass of the system, $D$ is the mechanical damping of the structure, $K_{S C}$ is the shortcircuited stiffness, $C_{p}$ is the piezoelectric capacitance, and $\alpha$ is the electromechanical coupling between the mechanical part and the electrical part of the piezoelectric generator. 


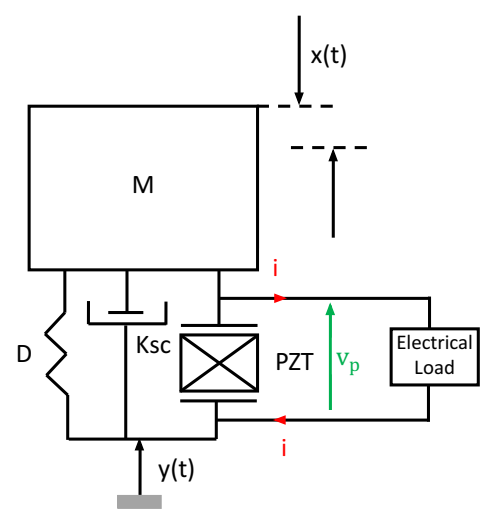

Fig. 2. Generic mechanical-to-electrical conversion model

\section{Normalized power expression induced by the electrical load influence}

In the case of highly coupled systems, the electrical load has not only an influence on the electrical damping, but also on the electrical stiffness which can be used to tune the resonant frequency of the generator. In order to describe this effect, we first need to define the piezoelectric voltage as a combination of an imaginary part (the normalized electrical damping $\epsilon_{D}$ ) and a real part (the normalized electrical stiffness $\epsilon_{K}$ ). These parameters imply there is a phase difference between the piezoelectric voltage $v_{p}$ and dynamic mass displacement $x$. In order to express the piezoelectric voltage consequently, we use the following notation defined in (Badel and Lefeuvre, 2016) and the following equivalent circuit:

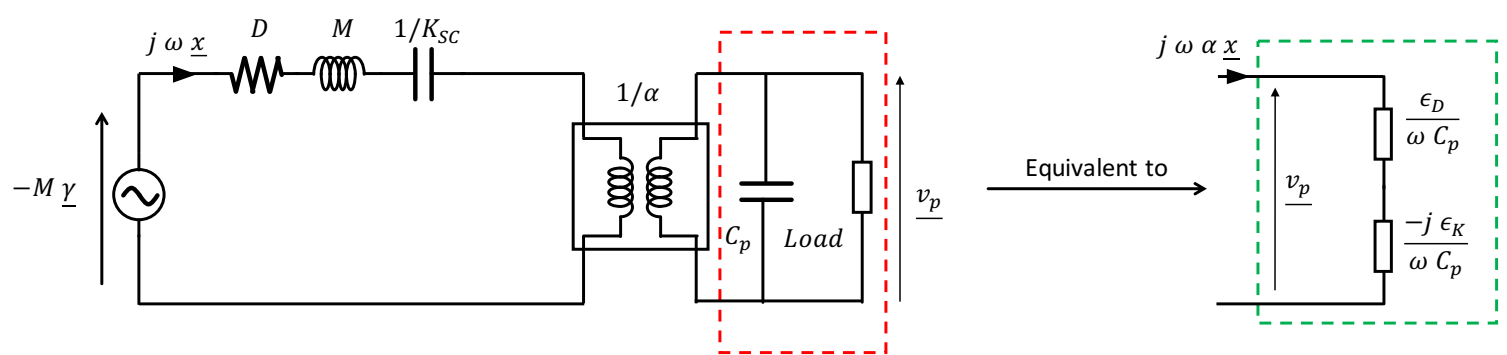

Fig. 3. Equivalent circuit of a PEH and its electrical load

$$
\underline{v_{p}}=\frac{\alpha \underline{x}}{C_{p}}\left(\epsilon_{K}+j \epsilon_{D}\right)
$$

Injecting (2) in (1) written in the frequency domain leads to the following expression of the relative displacement $\underline{x}$ : 


$$
\underline{x}=\frac{-M \underline{\gamma}}{\left(K_{s c}+\frac{\alpha^{2}}{C_{p}} \epsilon_{K}-M \omega^{2}\right)+j\left(\omega D+\frac{\alpha^{2}}{C_{p}} \epsilon_{D}\right)}
$$

$\underline{x}$ can also be expressed as the following expression using normalized constants:

$$
\underline{x}=\frac{\gamma}{\left(1-\Omega_{e m}^{2}\right)+2 j \Omega_{m}\left(\xi_{m}+\xi_{e}\right)} \text { with }\left\{\begin{array}{c}
\omega_{0}=\sqrt{\frac{K_{s c}}{M}} \\
\xi_{m}=\frac{D}{2} \sqrt{\frac{1}{K_{s c} M}} \\
\xi_{e}=\frac{k_{m}^{2} \epsilon_{D}}{2 \Omega_{m}} \\
\Omega_{m}=\frac{\omega}{\omega_{0}} \\
\Omega_{e m}^{2}=\Omega_{m}^{2}-\Omega_{e} \\
\Omega_{e}=k_{m}^{2} \epsilon_{K} \\
k_{m}^{2}=\frac{k^{2}}{1-k^{2}}=\frac{\alpha^{2}}{K_{S C} C_{p}}
\end{array}\right.
$$

Where $\omega_{0}$ is the short circuit mechanical resonant frequency, $\xi_{m}$ and $\xi_{e}$ are the normalized damping ratios due to mechanical losses and electrical resistance respectively, and $\Omega_{m}$ is the normalized (with respect to $\omega_{0}$ ) short circuit mechanical resonant frequency. $\Omega_{e m}$ is the normalized resonant frequency of the electromechanical system, taking into account both the mechanical part and electrical part of the generator. $\Omega_{e}$ is defined as the resonant frequency variation due to the electrical stiffness. $k^{2}$ is the squared coupling coefficient, and $k_{m}{ }^{2}$ is defined as the squared modified electromechanical coupling coefficient, and is a function of the coupling factor $\alpha$, the piezoelectric capacity $C_{p}$ and the short-circuit stiffness $K_{S C}$.

Normalizing $\underline{x}$ in (4) with respect to $\frac{\gamma}{\omega_{0}^{2}}$ leads to the following expression:

$$
\underline{\tilde{x}}=\frac{-1}{\left(1-\Omega_{e m}^{2}\right)+2 j \Omega_{m}\left(\xi_{m}+\xi_{e}\right)}
$$

From (5), it can be clearly seen that the resonance frequency is reached when $\Omega_{e m}$ is equal to 1 . It means that the resonant frequency of the system is not only dependent of 
the mechanical properties of the harvester, but is also tunable by the electrical stiffness of the load. The harvested power is the power dissipated in the electrical damper $\left(\frac{\epsilon_{D}}{\omega C_{p}}\right)$ i.e. in the real part of the load, as shown in Fig. 3. It can be expressed as:

$$
P=\frac{\omega \alpha^{2} \epsilon_{D} \underline{x}_{\max }^{2}}{2 C_{p}}=\frac{\gamma^{2} \xi_{e} M \Omega_{m}^{2}}{\omega_{0}\left(\left(1-\Omega_{e m}^{2}\right)^{2}+4 \Omega_{m}^{2}\left(\xi_{m}+\xi_{e}\right)^{2}\right)}
$$

\section{Optimal electrical damping}

By finding the roots of the derivative of (6) with respect to $\xi_{e}$, we can express the optimized electrical damping $\xi_{e_{\text {opt }}}$ that maximizes the power at the resonance and off resonance:

$$
\xi_{\text {opt }}=\frac{\sqrt{\left(\Omega_{m}^{2}-\Omega_{e}\right)^{2}+4 \Omega_{m}^{2} \xi_{m}^{2}-2 \sqrt{\Omega_{m}^{2}-\Omega_{e}}+1}}{2 \Omega_{m}}
$$

From equation (7), we can easily see that when the vibration frequency matches the normalized resonant frequency of the electromechanical system $\left(\Omega_{e m}=1\right)$, the optimized electrical damping is equal to the mechanical damping. However, when the input frequency is different than the system's resonant frequency, the optimized electrical damping is greater than the mechanical damping. If $\Omega_{e} \neq 0\left(\Omega_{e m} \neq \Omega_{m}\right)$, the optimized power (and minimum optimal damping) is reached for a $\Omega_{m}$ different than 1, which means that the resonant frequency of the system can be tuned using this electrical stiffness.

The power is maximized when the optimal electrical damping is equal to the mechanical damping $\left(\xi_{e}=\xi_{m}\right)$ and when the vibration frequency matches the electromechanical system's resonant frequency $\left(\Omega_{e m}=1\right)$. The maximum power can then be expressed, as explained in (Badel and Lefeuvre, 2016):

$$
P_{\max }=\frac{M \gamma^{2}{ }_{\max }}{16 \omega_{0} \xi_{m}}=\frac{M^{2} \gamma^{2}{ }_{\max }}{8 D}
$$

In order to use a referenced value of the harvested power, we will be using the following expression in this paper, directly derived from (6) and (8): 


$$
P_{\text {norm }}=\frac{P Q}{P_{\max }}=\frac{8 \xi_{e} \Omega_{m}^{2}}{\left(1-\Omega_{e m}^{2}\right)^{2}+4 \Omega_{m}^{2}\left(\xi_{e}+\xi_{m}\right)^{2}}
$$

Using this notation, the power will be expressed as a number between 0 and $Q$ where $Q$ is the quality factor of the mechanical part of the harvester, and can be expressed as $Q=\frac{1}{2 \xi_{m}}$. $P_{\text {norm }}$ is only dependent of the electrical and mechanical damping and of the normalized frequencies $\Omega_{m}$ and $\Omega_{e m}$.

\section{Piezoelectric generator's admittance analysis}

In order to find the optimal impedance that maximizes the harvested power, it is important to analyze the piezoelectric generator's admittance. From (1), the piezoelectric generator's admittance can be expressed as:

$$
Y=j C_{p} \omega\left(1+\frac{k_{m}^{2}}{1-\Omega_{m}^{2}+2 j \xi_{m} \Omega_{m}}\right)
$$

The imaginary part of this admittance is useful in order to analyze the piezoelectric resonance frequencies and if the piezoelectric generator behaves as a capacitive generator or an inductive generator. It can be expressed as:

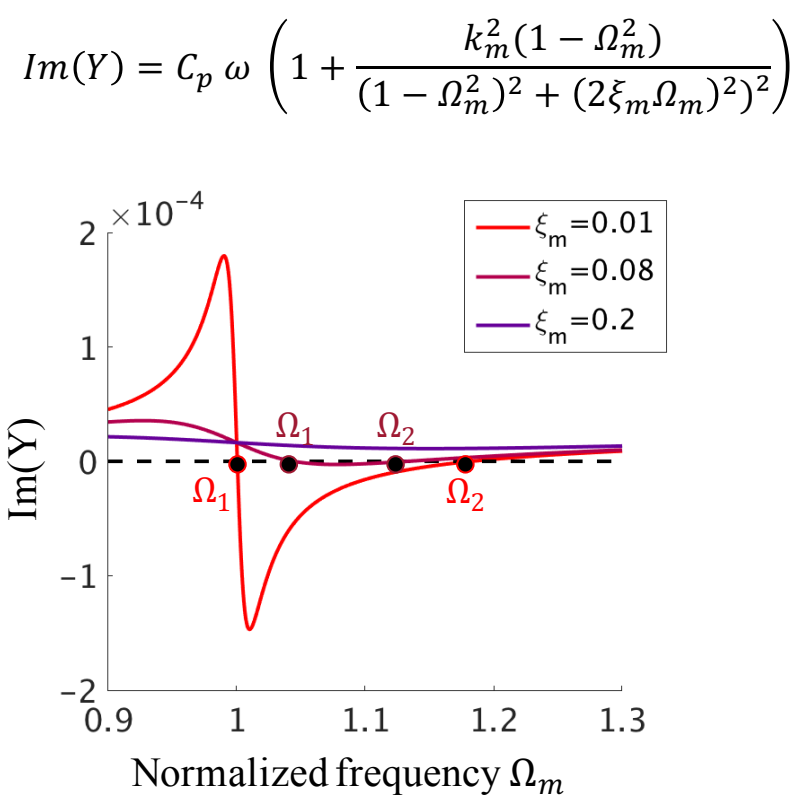

Fig. 4. Imaginary part of the admittance with different mechanical damping, with $\mathrm{k}_{\mathrm{m}}^{2}=0.4$ 
Fig. 4 shows the imaginary part of the admittance. The piezoelectric generator admittance exhibits one short-circuit resonance frequency and one open circuit resonance frequency (as explained later). Between these two frequencies, there is a range where the imaginary part of the admittance is negative, then the generator exhibits an inductive behavior. Otherwise, it is always positive. Fig. 4 also shows that these resonance frequencies are dependent on the mechanical damping. If the mechanical damping is too high, there may not be any resonance.

\section{High coupling influence on the admittance}

The two roots of (11) are named $\Omega_{1}$ and $\Omega_{2}$. For a highly coupled and lowly damped piezoelectric generator, $\Omega_{1}$ and $\Omega_{2}$ correspond to the short circuit and open circuit resonance normalized frequencies, respectively. In order to understand the influences of the damping and coupling on $\Omega_{1}$ and $\Omega_{2}$, we establish the following expressions:

$$
\left\{\begin{array}{c}
\Omega_{1}=\sqrt{\frac{2+k_{m}^{2}-4 \xi_{m}^{2}-\sqrt{\left(4 \xi_{m}^{2}-k_{m}^{2}\right)^{2}-16 \xi_{m}^{2}}}{2}} \approx 1 \\
\Omega_{2}=\sqrt{\frac{2+k_{m}^{2}-4 \xi_{m}^{2}+\sqrt{\left(4 \xi_{m}^{2}-k_{m}^{2}\right)^{2}-16 \xi_{m}^{2}}}{2}} \approx \sqrt{1+k_{m}^{2}}
\end{array}\right.
$$

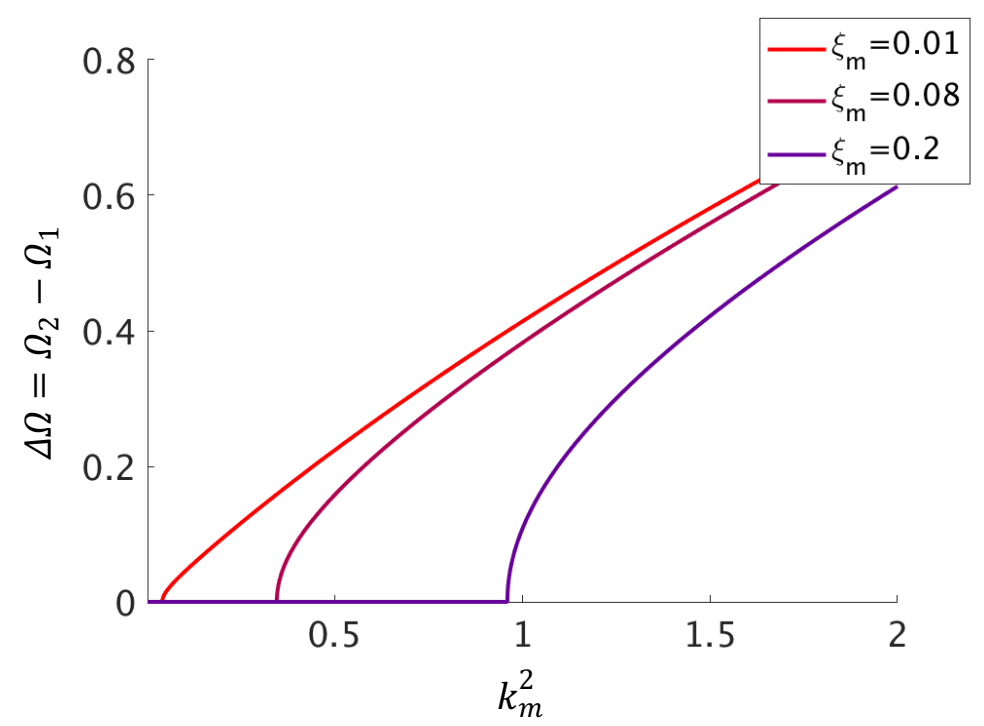

Fig. 5. Bandwidth between the anti-resonant frequency and resonant frequency as a function of $k_{m}^{2}$ and $\xi m$

If the damping is low enough, $\Omega_{1}$ will always be very close to 1 and will not be very affected by the coupling. However, $\Omega_{2}$ is strongly dependent on the coupling and will be shifted on the right as the coupling increases. As explained in (Badel and Lefeuvre, 2016), the damping limit is given by $k_{m}^{2} \approx 4 \xi_{m}$. If $k_{m}^{2}$ is below this value, the admittance is always strictly positive, and consequently, $\Omega_{1}$ and $\Omega_{2}$ are not defined. As it 
has already been shown in Fig. 4, the piezoelectric generator behaves like an inductive source for any frequency between $\Omega_{1}$ and $\Omega_{2}$. Hence the electrical load must be capacitive to achieve the maximum harvesting power. A higher coupling increases the width of the frequency band where the piezoelectric generator has an inductive behavior as shown in Fig. 5. This frequency range where the optimal load is inductor-less is given by (13).

$$
\Delta \Omega=\Omega_{2}-\Omega_{1} \approx \sqrt{1+k_{m}^{2}}-1
$$

\section{Influences of the reactive behaviors of the electrical interface on the harvested power}

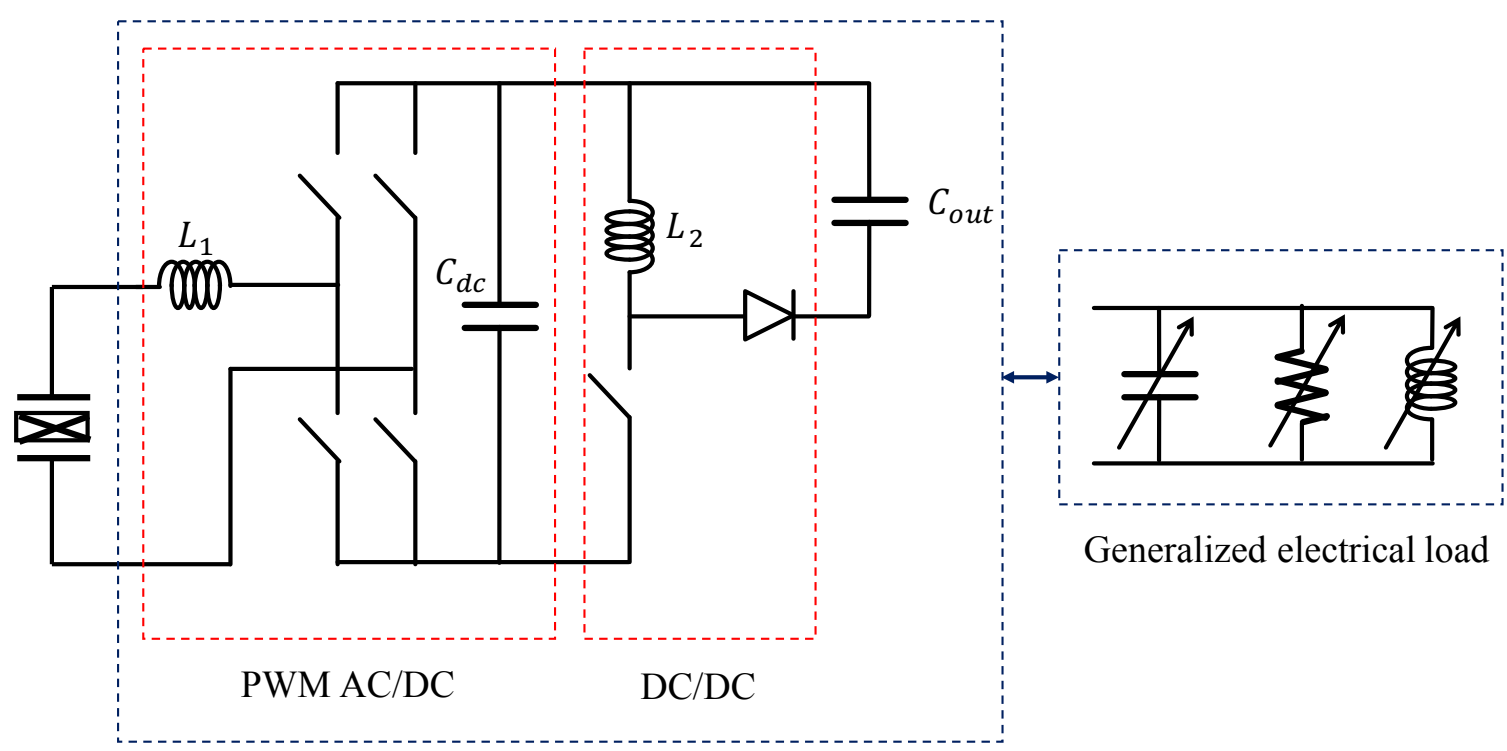

Fig. 6. Piezoelectric frequency and damping tuning using a generalized passive electrical load, emulated by a PWM rectifier.

Table 1

Examples of nonlinear strategies first-harmonic modelling

Interfacel

Equivalent impedance
$\varepsilon_{K}$ range (giving an indication of the ability of the strategy to emulate an important range of reactive loads)

\begin{tabular}{|c|c|c|}
\hline $\begin{array}{c}\mathrm{AC} / \mathrm{DC}+ \\
\mathrm{DC} / \mathrm{DC}\end{array}$ & $\frac{2 R_{d c} C_{p} \omega}{\left(R_{d c} C_{p} \omega+\pi / 2\right)^{2}}$ & $\frac{R_{d c} C_{p} \omega}{R_{d c} C_{p} \omega+\pi / 2}$ \\
\hline
\end{tabular}

SECE

$$
\frac{4(1+\gamma)}{2(1+\gamma) R_{d c} C_{p} \omega+\pi(1-\gamma)}
$$


Regenerative
SECE

Phase-shifted SECE

Frequency

Tuning SECE

$$
\frac{2\left[1-(-1)^{N}\right]}{\pi N}
$$

$$
\frac{4}{\pi} \cos ^{2}(\phi)
$$

$$
\begin{gathered}
1+\frac{2}{\pi} \sin (\phi) \\
1+\frac{2}{\pi} \frac{(1-\beta)}{(1+\beta)} \sin (2 \phi)
\end{gathered}
$$

$\frac{4}{\pi} \frac{(1-\beta)}{(1+\beta)} \cos ^{2}(\phi)$

As proven in prior art, all the electrical interfaces can be modelled, thanks to the first harmonic approximation, as a combination of resistive and reactive impedances (Liang and Liao, 2010, Badel and Lefeuvre, 2016), as shown in Fig. 6. We can note that the PWM AC/DC circuit shown in Fig. 6 and presented in (Badel and Lefeuvre, 2016), is a possible way to emulate every combination of RLC loads and to realize a complex impedance matching. This means that, practically, the optimal impedance matching is realizable. However, the sophisticated algorithm as well as the switching losses associated with such circuit may consume a lot of energy, which hinder its potential use as an energy harvester interface. In order to find less consuming ways to emulate reactive loads, (Morel et al., 2018; Lefeuvre et al., 2017, Badel and Lefeuvre, 2014) have proposed alternative strategies, summarized in Table 1 . These strategies have been modelled using the formalism presented in this paper. $R_{d c}, \gamma, N, \beta$ and $\phi$ represent the input impedance of the $\mathrm{DC} / \mathrm{DC}$ converter which follows the $\mathrm{AC} / \mathrm{DC}$ converter, the charges inversion factor, the number of semi-period between every harvesting event, the percentage of electrical charges that are inverted during the energy harvesting event, and the angular phase between the displacement extremum and the energy harvesting event, respectively. Thus, in this part, we study the different influences involved with resistive, capacitive and inductive electrical behaviors. First, we investigate the harvested power using a pure resistive load. It aims at verifying the $P_{\max }$ expression given in (8). In a second time, we add a capacitive load in parallel to the resistive load in order to validate the possibility to tune the resonant frequency of the system and harvest the maximum power between $\Omega_{1}$ and $\Omega_{2}$. In a last part, we add an inductive load in parallel to the capacitance and resistance in order to study the performances of the harvester on a tuned RLC load to ideally maximize theoretically the harvested power for any vibration frequencies.

\section{Resistive electrical interface}

From equations (1) and considering a purely resistive electrical interface, the following equations that express the relation between the piezoelectric voltage and the current in the resistive load are derived. 


$$
\left\{\begin{array}{c}
i(t)=\alpha \dot{x}(t)-C_{p} \dot{v}_{p}(t) \\
i(t)=\frac{v_{p}(t)}{R}
\end{array}\right.
$$

Solving this system in the frequency domain leads to the following system:

$$
\left\{\begin{array}{c}
\underline{v_{p}}=\frac{\alpha}{C_{p}} \underline{x}\left(\epsilon_{K}+j \epsilon_{D}\right) \\
\epsilon_{D}=\frac{r \Omega_{m}}{1+\left(r \Omega_{m}\right)^{2}} \\
\epsilon_{K}=\frac{\left(r \Omega_{m}\right)^{2}}{1+\left(r \Omega_{m}\right)^{2}} \\
r=R \omega_{0} C_{p}
\end{array}\right.
$$

Where $R$ is the resistive load, $r \in \mathbb{R}^{+}$is the corresponding normalized load, $\epsilon_{D}$ and $\epsilon_{K}$ the normalized electrical damping and stiffness respectively. Finding the root of the derivative of the electrical damping $\epsilon_{D}$ (15) proves that the maximum electrical damping emulated with a resistance is $1 / 2$ and that the corresponding resistance is given by (16).

$$
\max \left(\epsilon_{D}\right)=\frac{1}{2} \Leftrightarrow R=\frac{1}{C_{p} \omega_{0}}
$$

From (4) and (16), at the resonant frequency, we can extract the minimal electromechanical coupling that is necessary to obtain an electrical damping equals to the mechanical damping. The expression of this coupling factor is given by (17). For the following, we define a low coupling system as $k_{m}^{2}<k_{m_{l i m}}^{2}$.

$$
k_{m_{l i m}}^{2}=\frac{4 \xi_{m}}{1+4 \xi_{m}} \approx 4 \xi_{m}
$$

In low coupling systems $\left(k_{m}^{2}<k_{m_{l i m}}^{2}\right)$, the optimal electrical damping cannot be reached, which explains why the maximum power $P_{\text {max }}(8)$ is not attained (Lefeuvre et 
al., 2006). It is however possible, using highly nonlinear techniques, to emulate a higher electrical damping and hence being able to harvest the maximum power even when this condition is not respected (Lefeuvre et al., 2006). In high coupling systems $\left(k_{m}^{2}>\right.$ $\left.k_{m_{l i m}}^{2}\right)$, the optimal damping can be reached without using any synchronous strategies. In any cases, using (9) and (15), the normalized power harvestable on a pure resistance load can be expressed as the following:

$$
\left\{\begin{array}{c}
P_{R}=\frac{8 \xi_{e} \Omega_{m}^{2}}{\left(1-\Omega_{e m}^{2}\right)^{2}+4 \Omega_{m}^{2}\left(\xi_{m}+\xi_{e}\right)^{2}} \\
\Omega_{e m}^{2}=\Omega_{m}^{2}-k_{m}^{2} \epsilon_{K} \\
\xi_{e}=\frac{k_{m}^{2} \epsilon_{D}}{2 \Omega_{m}}
\end{array}\right.
$$

a.
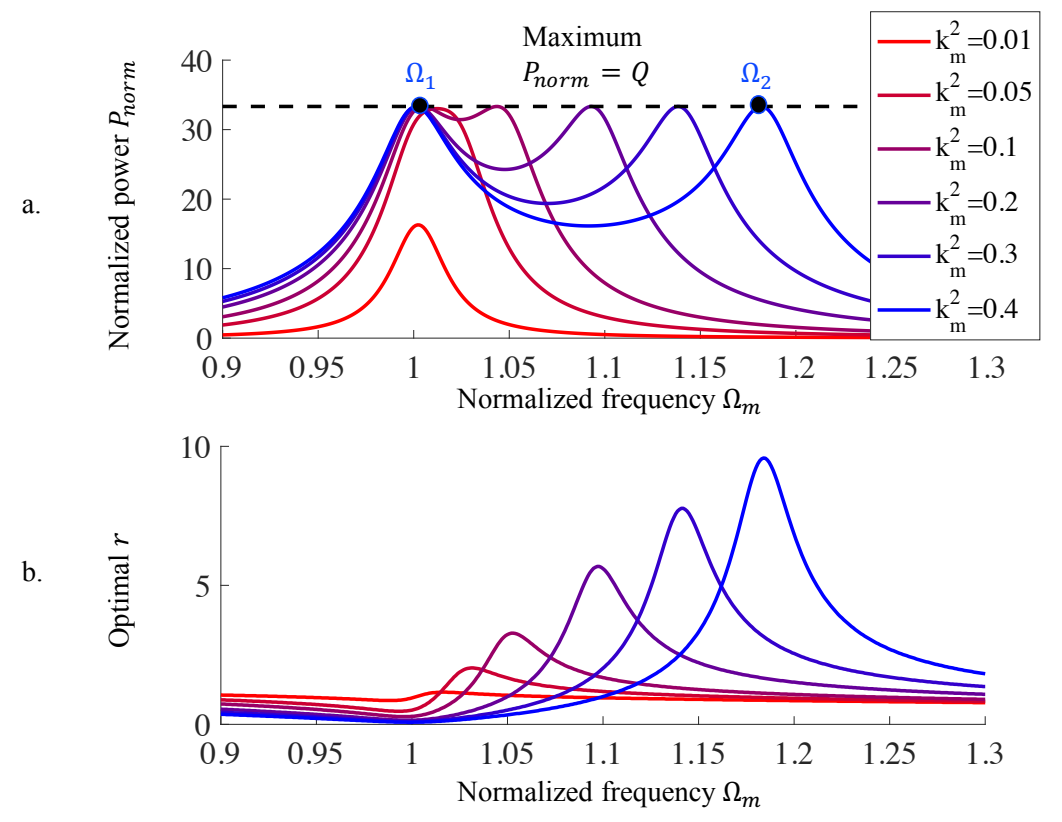

Fig. 7. (a) Maximum harvested power and (b) Optimal $r$ for harvesting on a purely resistive load with $\xi_{m}=0.015$

Finding the roots of the derivative of (18) leads to the optimal value of the normalized resistive load $r_{o p t}$, expressed by (19):

$$
r_{o p t}=\frac{1}{\Omega_{m}} \sqrt{\frac{\left(\Omega_{m}^{2}-1\right)^{2}+\left(2 \xi_{m} \Omega_{m}\right)^{2}}{\left(2 \xi_{m} \Omega_{m}\right)^{2}+\left(k_{m}^{2}+1-\Omega_{m}^{2}\right)^{2}}} \quad \forall \Omega_{m} \in \mathbb{R}^{+}
$$


The optimal normalized load $r$ for a particular mechanical damping $\left(\xi_{m}=0.015\right)$ leads to the results plotted in Fig. 7. It shows that the maximum harvested power is reached twice, for $\Omega_{m}=\Omega_{1}$ and the normalized frequency $\Omega_{m}=\Omega_{2}$ of the piezoelectric generator.

If the coupling is too small, $\left(k_{m}^{2}<k_{m_{l i m}}^{2}\right)$, the maximum power cannot be reached, since the electrical damping is too small to attain the mechanical damping. Hence in lowly coupled systems, the maximum harvested power depends on the coupling coefficient (Shu and Lien, 2006).

For highly coupled systems, the maximum power harvested at the resonance (and anti-resonance) is theoretically always equal to $P_{\max }$ as defined in (8). As shown in Fig. 5 , the open circuit resonance frequency is strongly dependent on the electromechanical coupling factor, which explains why the second peak $\Omega_{2}$ moves toward the right as the coupling is increased.

In order to enhance the bandwidth of the piezoelectric generator and to harvest the power limit $P_{\max }$ on a wide band of frequency, it should be possible to realize an impedance matching by using a capacitive load (since a highly coupled piezoelectric generator behaves as an inductive source between its short circuit and open circuit resonance frequencies) in order to maximize the harvested power between $\Omega_{1}$ and $\Omega_{2}$.

\section{Electrical interface combining resistive and capacitive behaviors}

Adding a capacitance in parallel to the resistive load is the same as increasing the dielectric capacitance of the piezoelectric generator. Increasing $C_{p}$ has the same effect as reducing the squared modified coupling coefficient $k_{m}^{2}$ since it is inversely proportional to $C_{p}(4)$. In order to study the power harvested by a RC load, we consider the system of equations (19), which takes into account the capacitive load $C$ and a normalized variable $c \in[0,1]$.

$$
\left\{\begin{array}{c}
\underline{v_{p}}=\frac{\alpha}{C_{p}} \underline{x}\left(\epsilon_{K}+j \epsilon_{D}\right) \\
\epsilon_{D}=\frac{r \Omega_{m}}{1+\left(\frac{r \Omega_{m}}{1-c}\right)^{2}} \\
\epsilon_{K}=\frac{\frac{\left(r \Omega_{m}\right)^{2}}{(1-c)}}{1+\left(\frac{r \Omega_{m}}{1-c}\right)^{2}} \\
r=R \omega_{0} C_{p} \\
c=\frac{C}{C+C_{p}}
\end{array}\right.
$$


Since the capacitive load $C$ only has an influence on the electrical damping and on the electrical stiffness, the global power expression remains the same as (18). Computing the maximum harvested power on a RC load gives the results exhibited in Fig. 8. For low coupling systems, the results are similar that those on a pure resistive load. We have shown that when $k_{m}^{2}<k_{m_{l i m}}^{2}$, the maximum power is an increasing function of the coupling factor. Hence, adding a capacitive load (which has the same effect as reducing the coupling) decreases the maximum harvested power.

In another hand, for highly coupled systems, the tuning of the capacitance (and hence the tuning of the normalized coupling) leads to a significant improvement of the harvested power between $\Omega_{1}$ and $\Omega_{2}$. Between these frequencies the piezoelectric generator exhibits an inductive behavior, hence the electrical load has to be capacitive. Another way to understand this tuning is to consider $\Omega_{2}$ as a moving frequency (function of the coupling factor) as seen in Fig. 5. At this frequency, the maximum harvested power can be reached. Hence, tuning the load capacity amount by adapting the normalized coupling, it is then possible to move this anti-resonant peak in order to harvest a maximized power between the resonant frequency and the anti-resonant frequency (which corresponds to the maximum reachable coupling). These results are consistent with the results obtained in (Ahmed-Seddik et al., 2013) that show that adding capacitances in parallel with the piezoelectric material tune the stiffness and hence the resonance of the harvesting system.

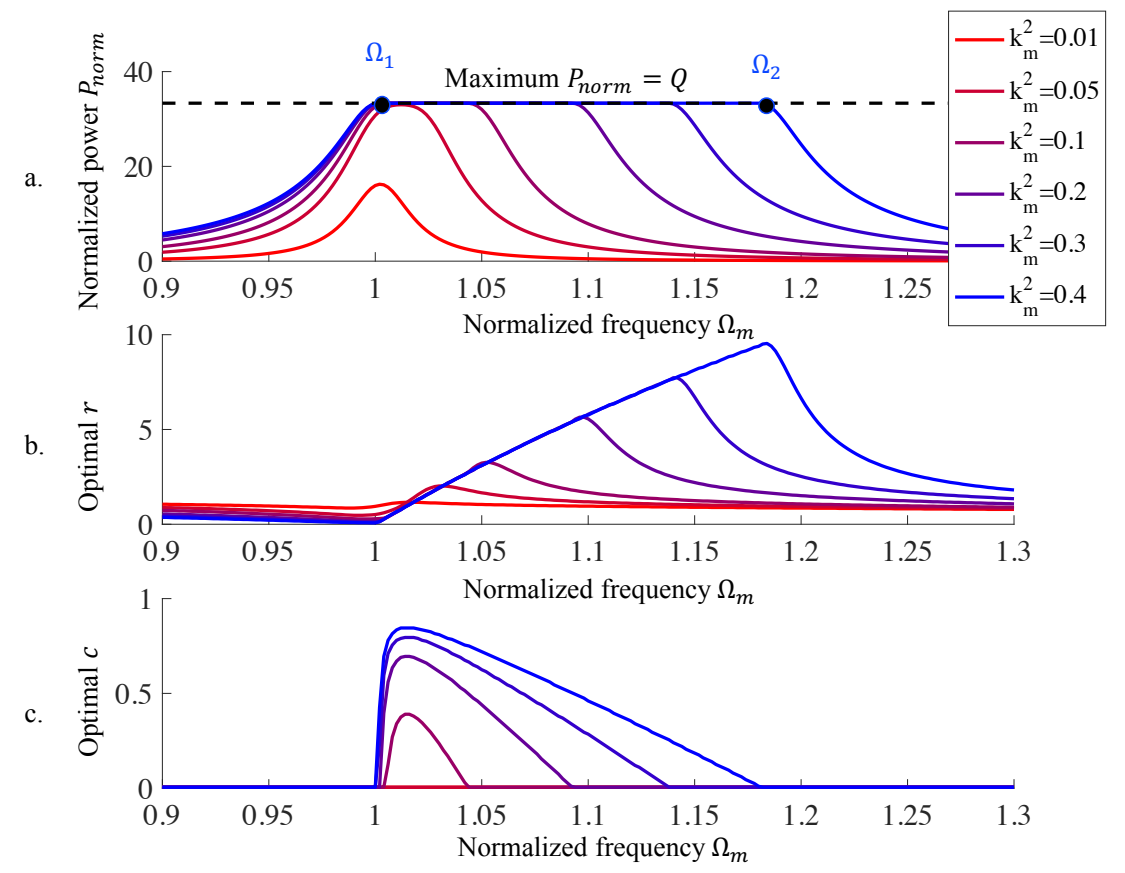

Fig. 8. (a) Maximum harvested power, (b) Optimal $r$ and (c) Optimal $c$ for harvesting on a RC load with $\xi_{m}=0.015$ 
Since the piezoelectric harvester works at resonance between $\Omega_{1}$ and $\Omega_{2}$, the analytical expression of the optimal electrical damping on this frequency band is directly equal to the mechanical damping. It leads to the followings optimal load expressions, valid for lowly damped/highly coupled systems:

$$
\begin{gathered}
\left.\left.\forall \Omega_{m} \in\right] 0, \Omega_{1}\right] \cup\left[\Omega_{2}, \infty\left[,\left\{\begin{array}{c}
r_{o p t}=\frac{1}{\Omega_{m}} \sqrt{\frac{\left(\Omega_{m}^{2}-1\right)^{2}+\left(2 \xi_{m} \Omega_{m}\right)^{2}}{\left(2 \xi_{m} \Omega_{m}\right)^{2}+\left(k_{m}^{2}+1-\Omega_{m}^{2}\right)^{2}}} \\
c_{o p t}=0
\end{array}\right.\right.\right. \\
\left.\forall \Omega_{m} \in\right] \Omega_{1}, \Omega_{2}\left[,\left\{\begin{array}{c}
r_{o p t}=\frac{\left(\Omega_{m}^{2}-1\right)^{2}}{2 k_{m}^{2} \Omega_{m}^{2} \xi_{m}}+\frac{2 \xi_{m}}{k_{m}^{2}} \\
c_{o p t}=1-\frac{\left(2 \Omega_{m} \xi_{m}\right)^{2}}{k_{m}^{2}\left(\Omega_{m}^{2}-1\right)}-\frac{\Omega_{m}^{2}-1}{k_{m}^{2}}
\end{array}\right.\right.
\end{gathered}
$$

Electrical interface combining resistive, capacitive and inductive behaviors

In this part we investigate how combining an inductive load with a capacitive load can enlarge the frequency band where $P_{\max }$ is harvested. We need to redefine the piezoelectric voltage and the normalized load expressions. Starting from (14), we derive the following expressions for a RLC load:

$$
\left\{\begin{array}{c}
\underline{v_{p}}=\frac{\alpha}{C_{p}} \underline{x}\left(\epsilon_{K}+j \epsilon_{D}\right) \\
\epsilon_{D}=\frac{r \Omega_{m}}{1+\left(\frac{r}{\Omega_{m} l^{2}}-\frac{r \Omega_{m}}{(1-c)}\right)^{2}} \\
\epsilon_{K}=\frac{\left(-\frac{r^{2}}{l^{2}}+\frac{r^{2} \Omega_{m}^{2}}{(1-c)}\right)}{1+\left(\frac{r}{\Omega_{m} l^{2}}-\frac{r \Omega_{m}}{(1-c)}\right)^{2}} \\
r=R \omega_{0} C_{p} \\
c=\frac{C}{C+C_{p}} \\
l=\omega_{0} \sqrt{L C_{p}}
\end{array}\right.
$$

Where $L$ is the inductive load, $r, c$ and $l$ are respectively the normalized load linked to the resistive, capacitive and inductive loads, and $\epsilon_{D}$ and $\epsilon_{K}$ are respectively the 
normalized electrical damping and normalized electrical stiffness emulated by the electrical load. Deriving $\epsilon_{D}(22)$ with respect to $l$, and studying its root shows that the maximum damping is directly proportional to $r$. It means that the inductive load can compensate the influence of the dielectric capacitance in order to make the electrical damping an unbounded function, proportional to the resistive load $R$ :

$$
\max \left(\epsilon_{D}\right)=r \Omega_{m} \Leftrightarrow L=\frac{1}{\left(C_{p}+C\right) \omega^{2}}
$$

Combining (18) and (22), the harvested power in the RLC load can be expressed. Finding numerically the maximum extracted power on a RLC load leads to Fig. 9. Outside $\left[\Omega_{1}, \Omega_{2}\right]$, the piezoelectric generator is capacitive (as shown in Fig. 4), hence it is understandable that the electrical load should be inductive on this frequency band. Between the resonant and anti-resonant frequency, the maximized power can be harvested without any inductance and using a capacitive tuning. The normalized expressions of the optimal loads can be expressed as follow:

$$
\begin{aligned}
& \left.\left.\forall \Omega_{m} \in\right] 0, \Omega_{1}\right] \cup\left[\Omega_{2}, \infty\left[,\left\{\begin{array}{c}
r_{o p t}=\frac{\left(\Omega_{m}^{2}-1\right)^{2}}{2 k_{m}^{2} \Omega_{m}^{2} \xi_{m}}+\frac{2 \xi_{m}}{k_{m}^{2}} \\
c_{o p t}=0
\end{array}\right.\right.\right. \\
& \left.\forall \Omega_{m} \in\right] \Omega_{1}, \Omega_{2}\left[,\left\{\begin{array}{c}
r_{o p t}=\frac{\left(\Omega_{m}^{2}-1\right)^{2}}{2 k_{m}^{2} \Omega_{m}^{2} \xi_{m}}+\frac{2 \xi_{m}}{k_{m}^{2}} \\
c_{o p t}=1-\frac{\left(2 \Omega_{m} \xi_{m}\right)^{2}}{k_{m}^{2}\left(\Omega_{m}^{2}-1\right)}-\frac{\Omega_{m}^{2}-1}{k_{m}^{2}} \\
l_{o p t}=\infty
\end{array}\right.\right.
\end{aligned}
$$

The inductances required to harvest a maximized energy outside of the resonant and anti-resonant frequencies are usually huge. For example, to harvest the maximum achievable power for the piezoelectric generator described in the next section and illustrated in Fig. 10, and driven $10 \%$ under its resonant frequency $\left(\Omega_{m}=0.9\right)$, a $247 \mathrm{H}$ inductance would be required. This value cannot be practically obtained using discrete components. However, as explained in (Abdelmoula and Abdelkefi, 2015), it is possible to use an interface emulating an inductive behavior in order to reach such value. This interface could be for example the PWM structure shown in Fig. 6 and proposed in (Badel and Lefeuvre, 2016), that tunes the phase between the load current and the 
fundamental (first harmonic) voltage, and hence can emulate inductive or capacitive loads.

a.

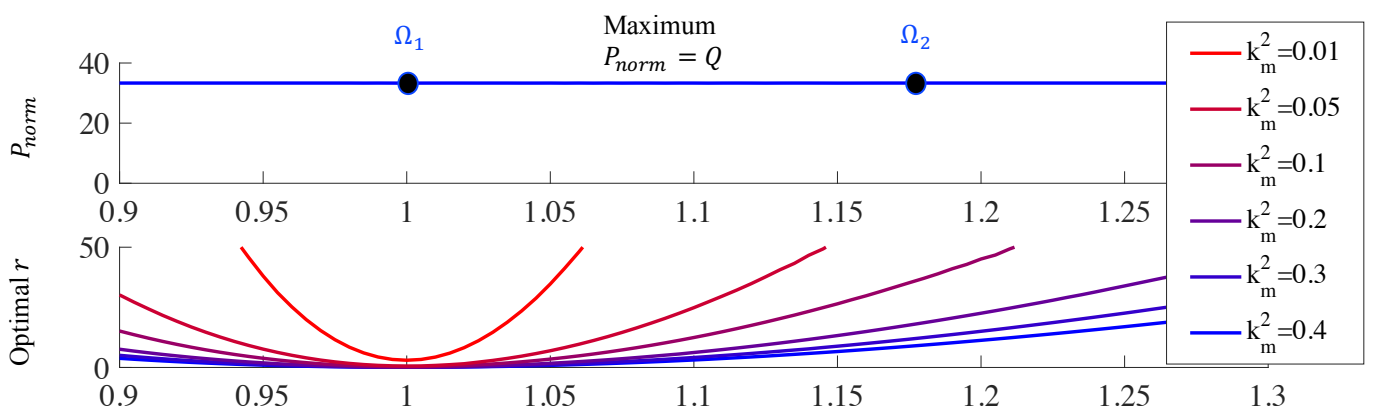

c.

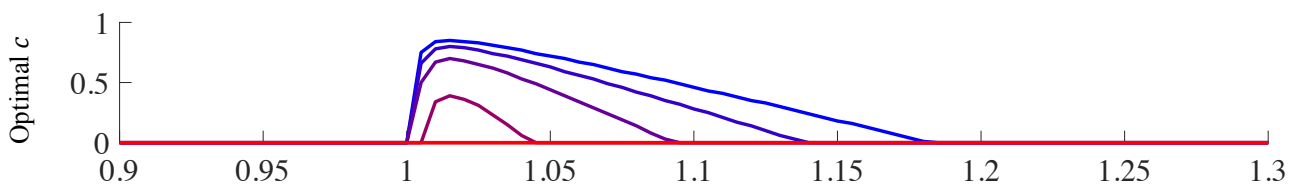

d.

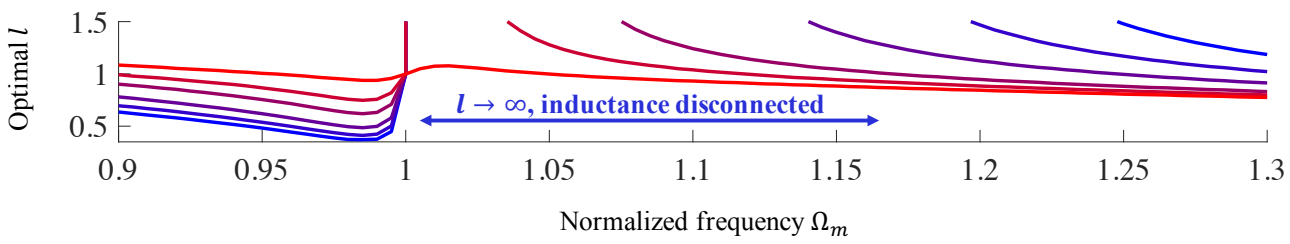

Fig. 9. (a) Maximum harvested power, (b) Optimal $r$, (c)c, and (d) $l$ for harvesting on a RLC load with $\xi_{m}=0.015$

\section{Experimental validation}

In this part, we validate the previously established theoretical results thanks to experimentations on a highly coupled piezoelectric generator, using a resistive load and a RC load alternately. Table 2 gives the characteristics of the piezoelectric generator used for the following experiments.

\section{Table 2}

Piezoelectric generator parameters table

\begin{tabular}{lcc}
\hline Parameters & Values & Units \\
\hline$k_{m}^{2}$ & 0.3 & - \\
$\xi_{m}$ & 0.01 & - \\
$C_{p}$ & 2.06 & $\mathrm{nF}$ \\
$f_{S C} \approx \frac{\Omega_{1} \omega_{0}}{2 \pi}$ & 98 & $\mathrm{~Hz}$ \\
$f_{O C} \approx \frac{\Omega_{2} \omega_{0}}{2 \pi}$ & 112 & $\mathrm{~Hz}$
\end{tabular}


The piezoelectric device consists in two plates of highly coupled piezoelectric material bonded on a cantilever beam, as shown in Fig. 10. The piezoelectric materials are PZN-PT single crystals that have been manufactured by Microfine Company. They exhibit an important coupling coefficient $k_{31}$ of 0.92 along the [100] axis. The whole experimental bench is illustrated in Fig. 11. The harvester is placed on an electrodynamic shaker. The cantilever tip displacement is sensed by a laser vibrometer and the vibration generated by the shaker by an accelerometer. The piezoelectric element is connected to a numerical resistive and a numerical capacitive decade box, and the piezoelectric voltage is displayed on an oscilloscope. Thanks to an algorithm implemented on dSpace, the voltage, displacement, and acceleration waveforms are stored in a computer for every load combination, and for various vibration's frequency.

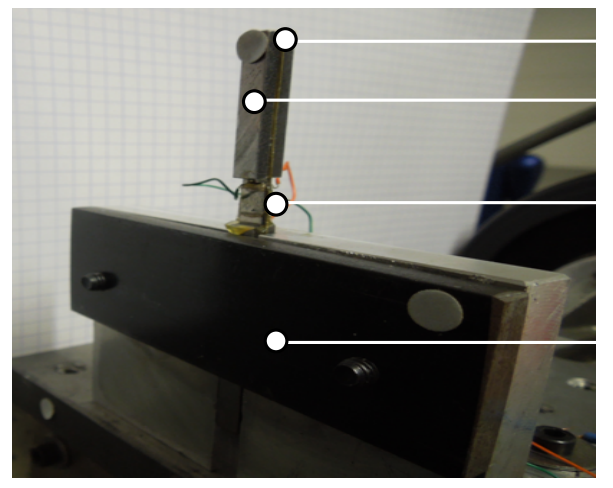

Cantilever beam, $40 \times 5 \times 0.3 \mathrm{~mm}^{3}$

2 Inertial masses in steel, $30 \times 5 \times 2.5 \mathrm{~mm}^{3}$

2 PZN-PT plates, $10 \times 5 \times 0.5 \mathrm{~mm}^{3}$

Support

Fig. 10. Photograph of the piezoelectric energy harvester exhibiting an important electromechanical coupling.

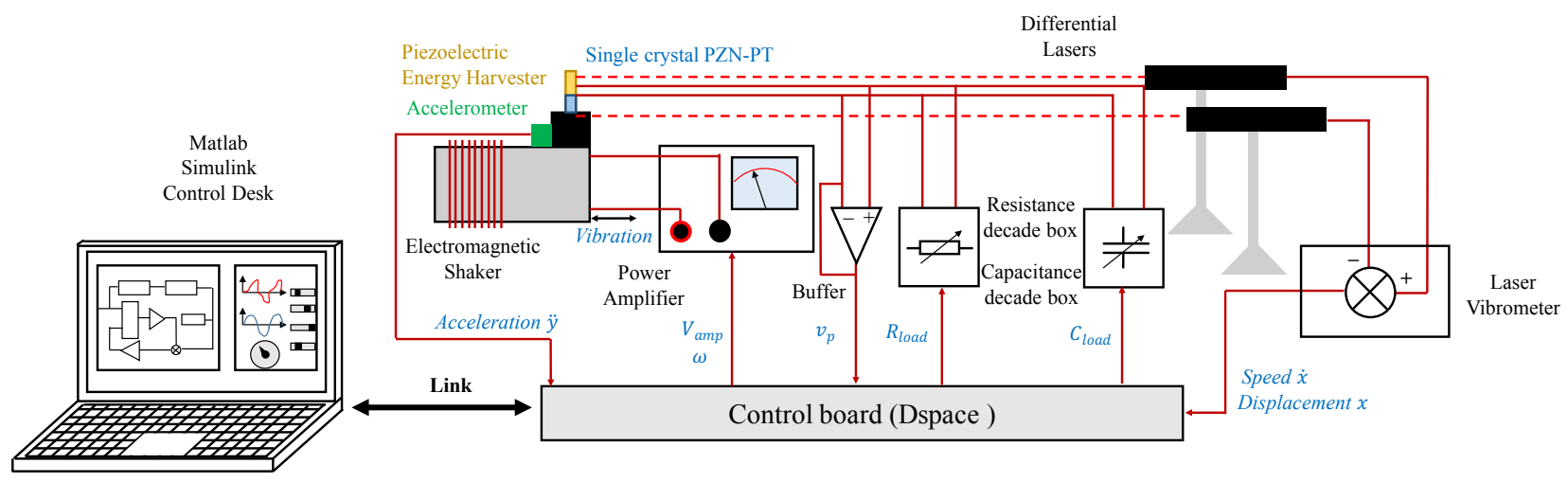

Fig. 11. Experimental setup 


\section{Results and discussion}

To verify the theoretical results, we compared the results of expression (18) applied on the piezoelectric generator using the characteristics listed in Table 2, and the results obtained through experimental tests under an acceleration of constant amplitude $\gamma_{\max }=0.5 \mathrm{~m} . \mathrm{s}^{-2}$. The results on a resistive load can be observed in Fig. 12, for 50 resistive loads values from $1 \mathrm{k} \Omega$ to $15 \mathrm{M} \Omega$, and 37 vibrations frequencies from $80 \mathrm{~Hz}$ to $135 \mathrm{~Hz}$. Fig. 12 shows the harvested power with the optimal resistance for each vibration's frequency. These results are in good agreements with the model and analysis done in the previous parts. The maximized power $(11 \mu \mathrm{W})$, as defined in (8), is attained at the short circuit resonance frequency $f_{s c}(98 \mathrm{~Hz})$ for a resistance of relatively small value $(60 \mathrm{k} \Omega)$. At the open circuit resonance frequency $f_{o c}(112 \mathrm{~Hz})$, there is another power peak corresponding to a higher resistive load value $(8 M \Omega)$, as predicted by the theoretical model and Fig. 7.

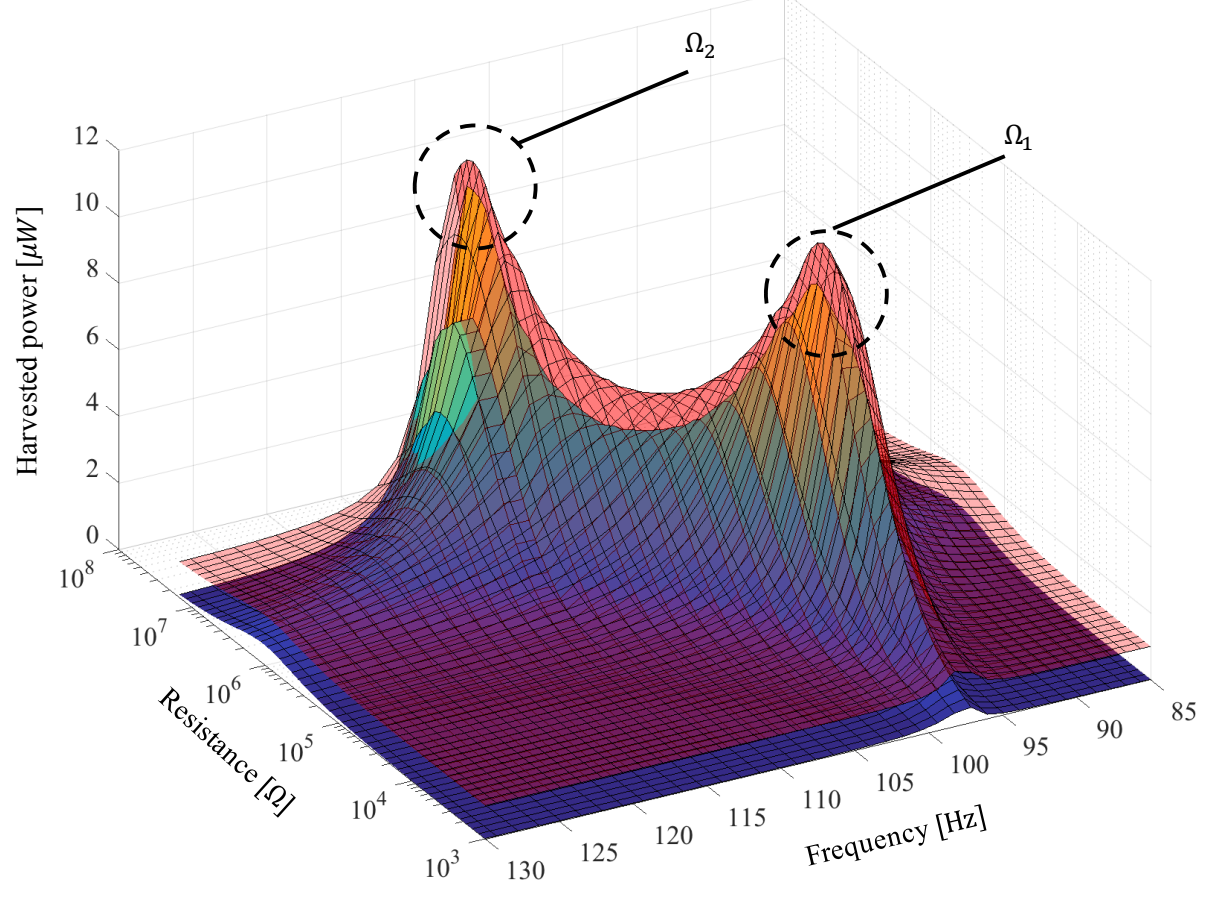

Fig. 12. Comparison between experimental (colored surface) and theoretical (transparent surface) harvested power on a resistive load in the $(R, f)$ plane. The theoretical surface has been shifted of $1 \mathrm{uW}$ in order to clarify the figure. 


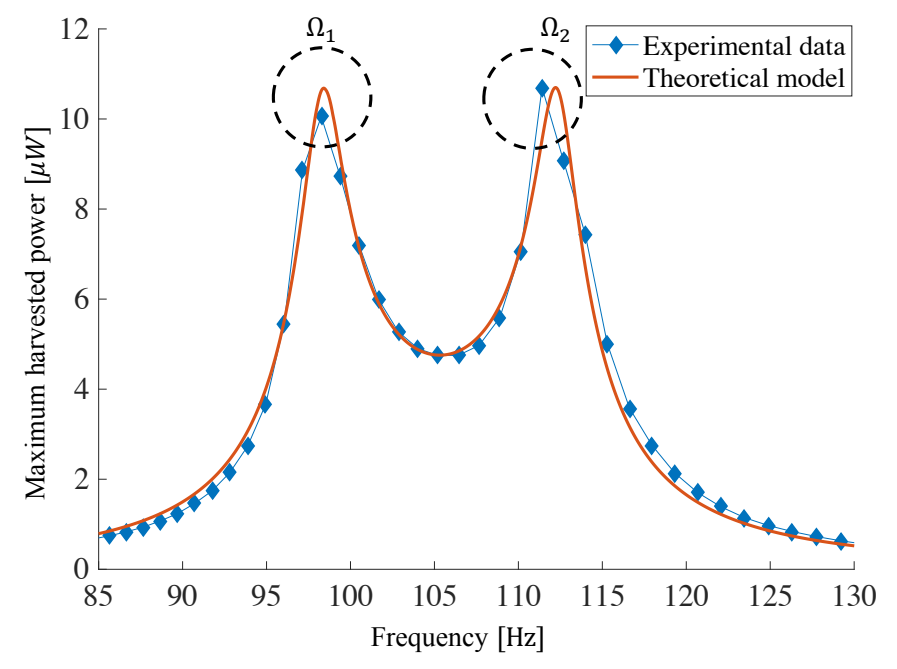

Fig. 13. Comparison between experimental (blue dots) and theoretical (red curve) maximum harvested power on an optimized resistive load

The influences of an additional capacitive load on the experimental harvester power response can be observed in Fig. 14, Fig.15 and Fig.16. As predicted from the theoretical analysis, the second power peaks $\left(\Omega_{2}\right)$ gets closer to the first power peak $\left(\Omega_{1}\right)$ as the load capacitance is increased. The maximum power however does not change, since the condition of high coupling given by (17) remains true.

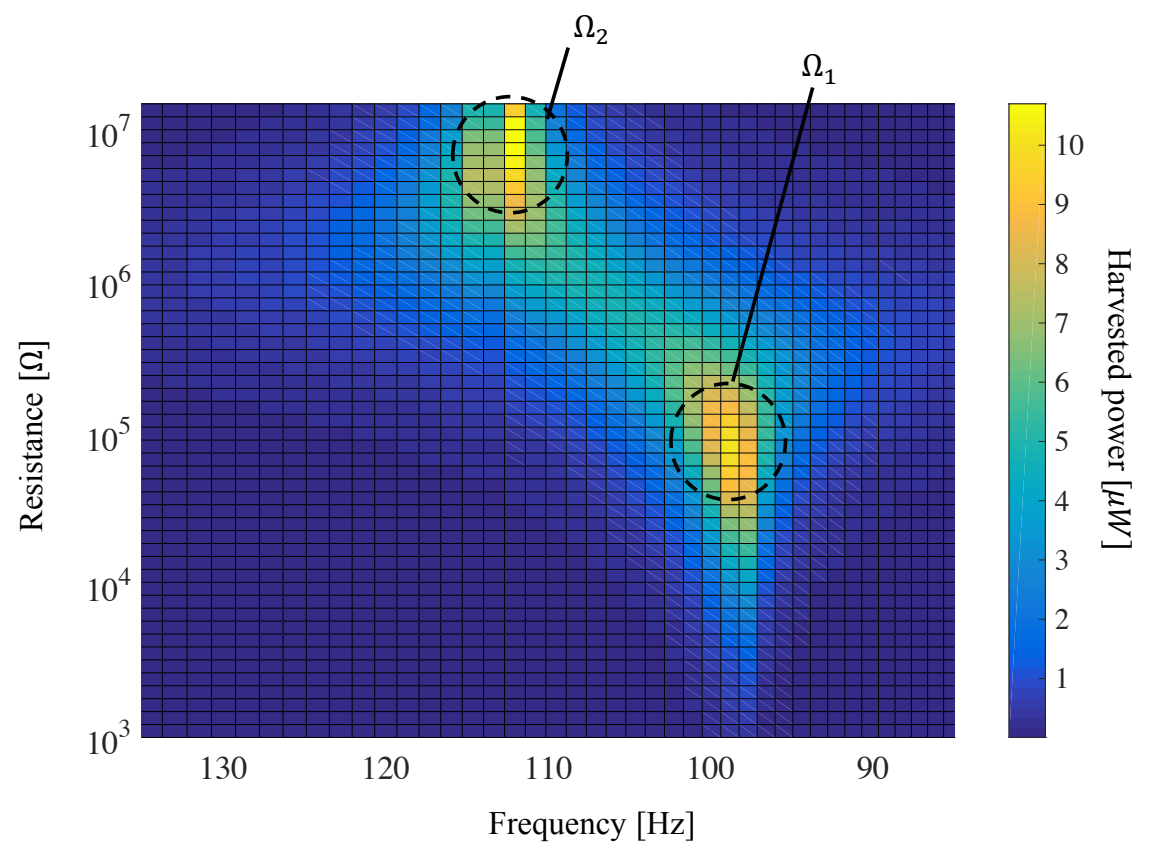

Fig. 14. Experimental harvested power in the $(R, f)$ plane with $C=0 n F$ 


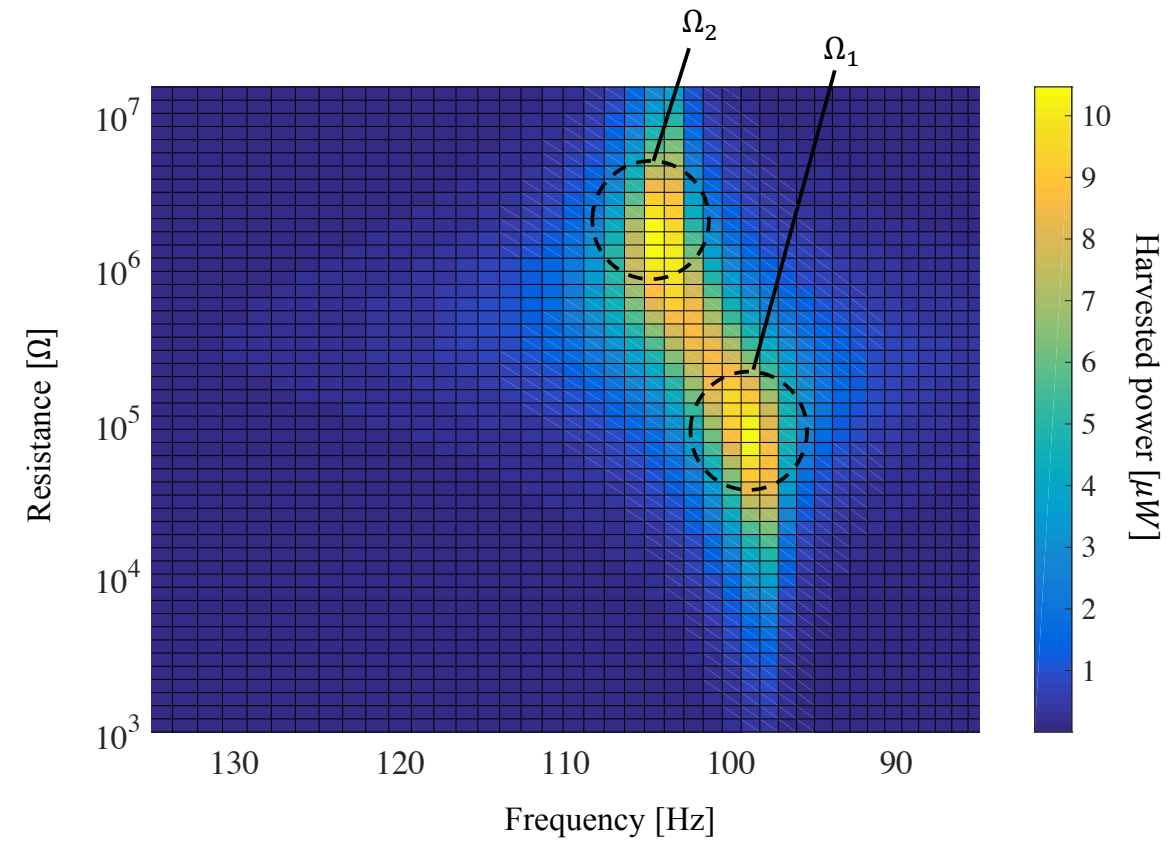

Fig. 15. Experimental harvested power in the $(R, f)$ plane with $C=3 n F$

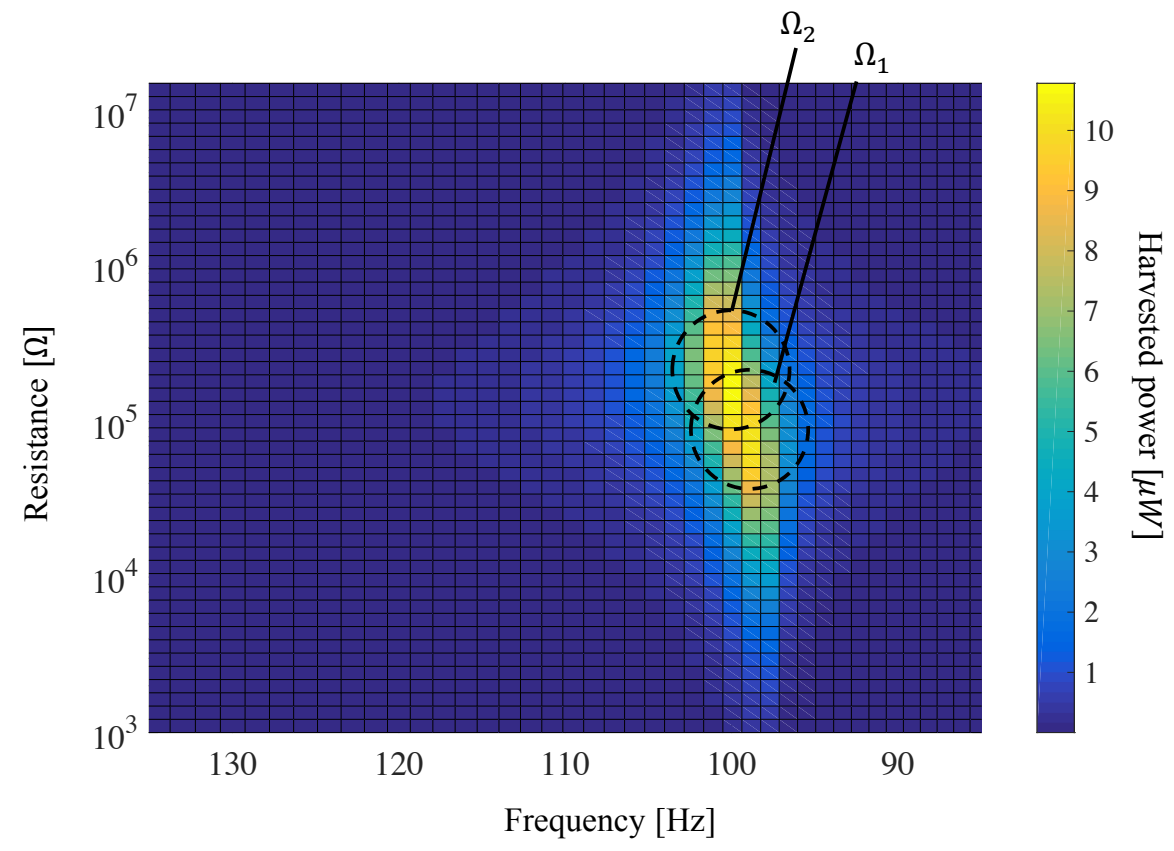

Fig. 16. Experimental harvested power in the $(R, f)$ plane with $C=11 n F$

The frequency responses of our PEH with optimized resistive loads and various capacitive loads have been measured as well, and are shown on Fig.17. 


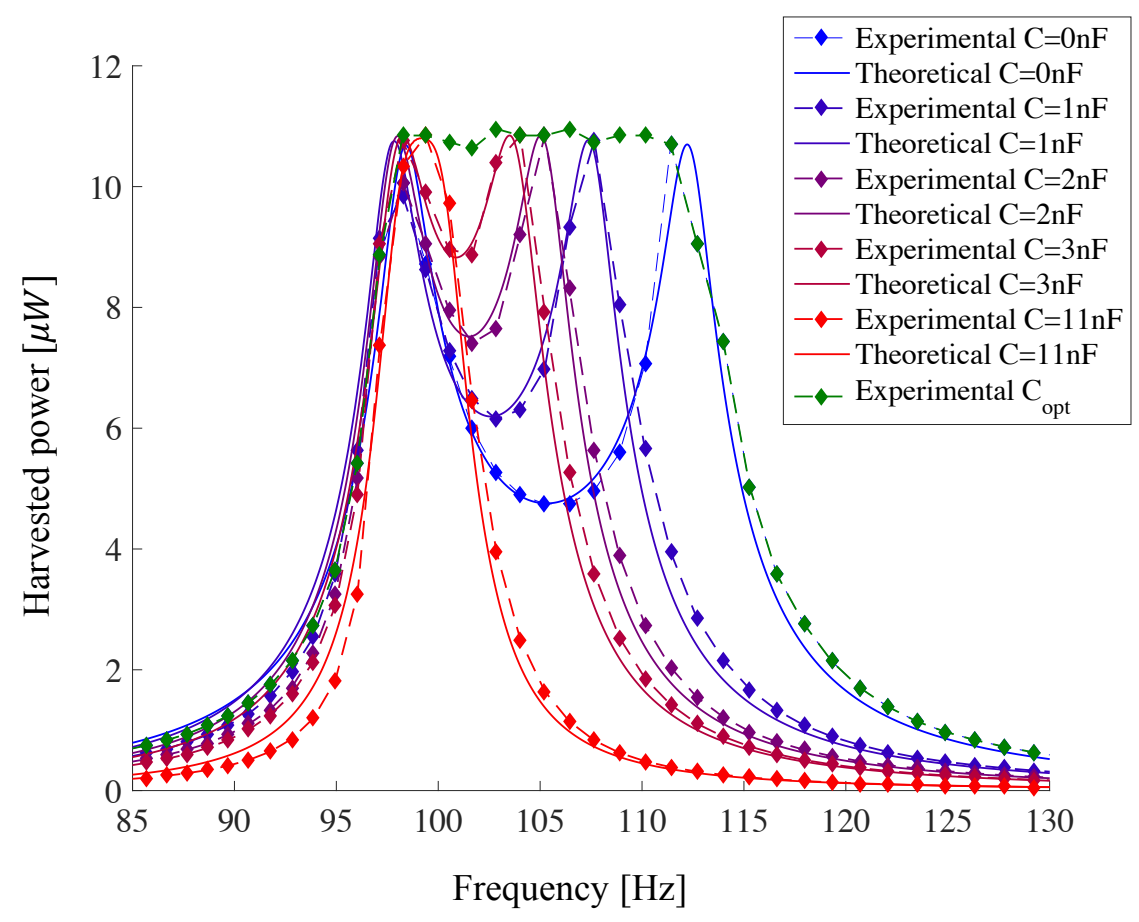

Fig. 17. Experimental (dots and dashed lines) and theoretical (straight lines) power frequency responses of our piezoelectric harvester with optimized resistive loads and various capacitive loads.

The experimental curves on Fig.17 look a lot like the theoretical ones on Fig.7. It confirms that adding a capacitance in parallel with the piezoelectric harvester tends to reduce its effective coupling and hence shifts the open circuit resonance frequency on the left. When adding a capacitance of $11 \mathrm{nF}$, the two power peaks become undistinguishable because the effective coupling is close to the limit coupling expressed by (17). If a higher capacitance value were added, the harvester would be lowly coupled, and the maximum harvested power would decrease. These results confirm that the harvested energy can be maximized between the resonant and anti-resonant frequency using a capacitive load that creates an electrical stiffness and tune the resonant frequency of the harvester. A fine tuning of the capacitive load (green curve on Fig. 17) allows to harvest the maximal power $(11 \mu W)$ for any frequency between $98 \mathrm{~Hz}$ and $112 \mathrm{~Hz}$, which are respectively the short circuit and open circuit resonance frequencies of the piezoelectric generator. This confirms the predicted behavior by Fig.8. The differences between the theoretical results and the experimental ones are relatively small and the relative error remains under $5 \%$.

\section{Conclusion}

In this paper, we develop the theoretical basis and notations that are required to extend the previous studies on linear harvesting approaches to highly coupled piezoelectric generators. We show the consequences of the increase of the piezoelectric electromechanical coupling factor on the harvesting strategies and optimal electrical load. A high coupling implies a large frequency band where the piezoelectric generator behaves as an inductive source. On this frequency band, it is possible to maximize the 
energy transfer by tuning an electrical stiffness using a capacitive load. These fundamental results have been demonstrated experimentally on a highly coupled PEH. The agreement between the theoretical results and the practical ones confirm that some new strategies and implementations could be investigated in order to realize not only a resistive emulation, but also a tunable capacitance matching.

Future work will aim at finding new strategies implementing an electrical stiffness tuning which could reach the theoretical limits presented in this paper. Continuous strategies focus on emulating capacitive tuning without any passive components, as proposed in (Morel et al., 2016), or using switching techniques such as Pulse Width Modulation (PWM) (Badel and Lefeuvre, 2016). Highly nonlinear strategies could also be used to control the energies exchange between the mechanical and electrical part, in order to emulate any inductive or capacitive values. The limitations of those all those strategies come from the frequency band where this tuning could be effectively done, the dielectric losses in the piezoelectric material (Liang and Liao, 2011; Morel et al., 2018) the losses induced by the switching (Badel and Lefeuvre, 2016) or by the quality factor of the inductance used in the inversion process (Badel and Lefeuvre, 2014). A potential aim could be to find new strategies that could overcome these limitations, while still emulating an important range of reactive loads.

\section{Funding}

The author(s) received no financial support for the research, authorship, and/or publication of this article.

\section{References}

Abdelmoula, H., Abdelkefi, A., 2015. Ultra-wide bandwidth improvement of piezoelectric energy harvesters through electrical inductance coupling. Eur. Phys. J. Spec. Top. 224, $2733-2753$.

Ahmed-Seddik, B., Despesse, G., Boisseau, S., et al. 2013. Self-powered resonant frequency tuning for Piezoelectric Vibration Energy Harvesters. J. Phys.: Conf. Ser. 476, 12069.

Arroyo, E., Badel, A., Formosa, F., et al. 2012. Comparison of electromagnetic and piezoelectric vibration energy harvesters: Model and experiments. Sensors and Actuators A: Physical 183, 148-156.

Badel, A., Lefeuvre, E., 2014. Wideband Piezoelectric Energy Harvester Tuned Through its Electronic Interface Circuit. J. Phys.: Conf. Ser. 557, 12115.

Badel. A., Lefeuvre, E., 2016. Nonlinear Conditioning Circuits for Piezoelectric Energy Harvesters. In Blokhina, E., Aroudi, A.E., Alarcon, E., Galayko, D., Nonlinearity Energy Harvest. Syst., Springer International Publishing, 2016, 321-359.

Brufau-Penella, J., Puig-Vidal, M., 2009. Piezoelectric Energy Harvesting Improvement with Complex Conjugate Impedance Matching. Journal of Intelligent Material Systems and Structures 20, 597-608.

Caliò, R., Rongala, U.B., Camboni, D., et al. 2014. Piezoelectric energy harvesting solutions. Sensors (Basel) 14, 4755-4790. 
Cho, J., Anderson, M., Richards, R., et al. 2005. Optimization of electromechanical coupling for a thin-film PZT membrane: I. Modeling. J. Micromech. Microeng. 15, 1797.

Guyomar, D., Badel, A., et al. 2005. Toward energy harvesting using active materials and conversion improvement by nonlinear processing. IEEE Transactions on Ultrasonics, Ferroelectrics, and Frequency Control 52, 584-595.

Guyomar, D., Lallart, M., 2011. Recent Progress in Piezoelectric Conversion and Energy Harvesting Using Nonlinear Electronic Interfaces and Issues in Small Scale Implementation. Micromachines 2, 274-294.

Hsieh, P., Chen, C., Chen, H., 2015. Improving the Scavenged Power of Nonlinear Piezoelectric Energy Harvesting Interface at Off-Resonance by Introducing Switching Delay. IEEE Transactions on Power Electronics 30, 3142-3155.

Lefeuvre, E., Badel, A., Brenes, A., et al. 2017. Power and frequency bandwidth improvement of piezoelectric energy harvesting devices using phase-shifted synchronous electric charge extraction interface circuit. Journal of Intelligent Material Systems and Structures 28, 2988-2995.

Lefeuvre, E., Badel, A., Richard, C., et al. 2005. Piezoelectric Energy Harvesting Device Optimization by Synchronous Electric Charge Extraction. Journal of Intelligent Material Systems and Structures 16, 865-876.

Lefeuvre, E., Badel, A., Richard, C., et al. 2006. A comparison between several vibrationpowered piezoelectric generators for standalone systems. Sensors and Actuators A: Physical 126, 405-416.

Lesieutre, G.A., Ottman, G.K., Hofmann, H.F., 2004. Damping as a result of piezoelectric energy harvesting. Journal of Sound and Vibration 269, 991-1001.

Liang, J., 2017. Synchronized bias-flip interface circuits for piezoelectric energy harvesting enhancement: A general model and prospects. Journal of Intelligent Material Systems and Structures 28, 339-356.

Liang, J., Liao, W.H., 2010. Impedance matching for improving piezoelectric energy harvesting systems, in: Active and Passive Smart Structures and Integrated Systems 2010 $7643,76430 \mathrm{~K}$.

Liang, J., Liao, W.H., 2011. On the Influence of Transducer Internal Loss in Piezoelectric Energy Harvesting with SSHI Interface. Journal of Intelligent Material Systems and Structures 22, 503-512.

Liao, Y., Sodano, H.A., 2009. Optimal parameters and power characteristics of piezoelectric energy harvesters with an RC circuit. Smart Mater. Struct. 18, 45011.

Morel, A., Badel, A., Wanderoild, Y., et al. 2018. A unified N-SECE strategy for highly coupled piezoelectric energy scavengers. Smart Materials and Structures, to be published.

Morel, A., Pillonnet, G., et al. 2018. Dielectric Losses Considerations for Piezoelectric Energy Harvesting, Journal of Low Power Electronics 14, to be published.

Morel, A., Grézaud, R., Pillonnet, G. et al. 2016. Active AC/DC control for wideband piezoelectric energy harvesting, J. Phys.: Conf. Ser. 773, 12059.

Ottman, G.K., Hofmann, H.F., Bhatt, A.C., et al. 2002. Adaptive piezoelectric energy harvesting circuit for wireless remote power supply. IEEE Transactions on Power Electronics 17, 669-676.

Raghunathan, V., Kansal, A., Hsu, J., et al. 2005. Design considerations for solar energy harvesting wireless embedded systems, in: IPSN 2005. Fourth International Symposium on Information Processing in Sensor Networks, 457-462. 
Schlichting, A., Tiwari, R., Garcia, E., 2012. Passive multi-source energy harvesting schemes. Journal of Intelligent Material Systems and Structures 23, 1921-1935.

Shi, G., Xia, Y., Ye, Y., et al. 2016. An efficient self-powered synchronous electric charge extraction interface circuit for piezoelectric energy harvesting systems. Journal of Intelligent Material Systems and Structures 27, 2160-2178.

Shu, Y.C., Lien, I.C., 2006. Efficiency of energy conversion for a piezoelectric power harvesting system. J. Micromech. Microeng. 16, 2429.

Shu, Y.C., Lien, I.C., 2006. Analysis of power output for piezoelectric energy harvesting systems. Smart Materials and Structures 15, 1499-1512.

Sodano, H.A., Simmers, G.E. et al. 2007. Recharging Batteries using Energy Harvested from Thermal Gradients. Journal of Intelligent Material Systems and Structures 18, 3-10.

Wanderoild, Y., et al. 2018. A $50 \mu \mathrm{W}$ Microbial Fuel Cell Isolated Energy Harvesting Interface Based on Air Coupled Inductors, Journal of Low Power Electronics 14, 170178.

Wu, W.-J., Chen, Y.-Y., Lee, B.-S., He, J.-J., Peng, Y.-T., 2006. Tunable resonant frequency power harvesting devices, in: Smart Structures and Materials 2006: Damping and Isolation. Presented at the Smart Structures and Materials 2006: Damping and Isolation, International Society for Optics and Photonics, p. 61690A.

Wu, Y., Badel, A., Formosa, F., et al. 2013. Piezoelectric vibration energy harvesting by optimized synchronous electric charge extraction. Journal of Intelligent Material Systems and Structures 24, 1445-1458.

Zhu, M., Worthington, E., Njuguna, J., 2009. Coupled piezoelectric-circuit FEA to study influence of a resistive load on power output of piezoelectric energy devices, in: Schmid, U. (Ed.), p. 736202. 